\title{
Inhibition of ATR potentiates the cytotoxic effect of gemcitabine on pancreatic cancer cells through enhancement of DNA damage and abrogation of ribonucleotide reductase induction by gemcitabine
}

\author{
SHUANG LIU ${ }^{1}$, YUBIN GE ${ }^{2,3}$, TINGTING WANG ${ }^{1}$, HOLLY EDWARDS $^{2,3}$, QIHANG REN $^{1}$, \\ YIQUN JIANG ${ }^{1}$, CHENGSHI QUAN ${ }^{4}$ and GUAN WANG ${ }^{1,4}$

\begin{abstract}
${ }^{1}$ National Engineering Laboratory for AIDS Vaccine, Key Laboratory for Molecular Enzymology and Engineering, The Ministry of Education, School of Life Sciences, Jilin University, Changchun, Jilin 130012, P.R. China;

${ }^{2}$ Department of Oncology, Wayne State University School of Medicine; ${ }^{3}$ Molecular Therapeutics Program, Barbara Ann Karmanos Cancer Institute, Wayne State University School of Medicine, Detroit, MI, USA;

${ }^{4}$ Department of Pathophysiology, College of Basic Medical Sciences, Jilin University, Changchun, Jilin 130021, P.R. China
\end{abstract}

Received September 23, 2016; Accepted March 31, 2017

DOI: $10.3892 /$ or.2017.5580

\begin{abstract}
Pancreatic cancer is a highly malignant disease with a dismal prognosis. Gemcitabine (GEM)-based chemotherapy is the first-line treatment for patients with advanced disease, although its efficacy is very limited, mainly due to drug resistance. Ataxia telangiectasia and Rad3-related (ATR) plays a critical role in the DNA damage response (DDR) which has been implicated in GEM resistance. Thus, targeting ATR represents a promising approach to enhance GEM antitumor activity. In the present study, we tested the antitumor activity of AZ20, a novel ATR-selective inhibitor, alone or combined with GEM in 5 pancreatic cancer cell lines. AZ20 treatment of the pancreatic cancer cell lines resulted in growth inhibition, with $\mathrm{IC}_{50}$ values ranging from 0.84 to $2.4 \mu \mathrm{M}$, but limited cell death. As expected, treatment of pancreatic cancer cell lines with AZ20 caused decreased phosphorylation of CHK1 (S-345). However, this was accompanied by DNA damage and $\mathrm{S}$ and $\mathrm{G} 2 / \mathrm{M}$ cell cycle arrest, independent of TP53 gene mutational status. Importantly, combination of AZ20 with GEM resulted in synergistic inhibition of cell growth and
\end{abstract}

Correspondence to: Dr Guan Wang, National Engineering Laboratory for AIDS Vaccine, Key Laboratory for Molecular Enzymology and Engineering, the Ministry of Education, School of Life Sciences, Jilin University, 2699 Qianjin Street, Changchun, Jilin 130012, P.R. China

E-mail:wg10@jlu.edu.cn

Dr Chengshi Quan, Department of Pathophysiology, College of Basic Medical Sciences, Jilin University, 126 Xinmin Street, Changchun, Jilin 130021, P.R. China

E-mail: quancs@jlu.edu.cn

Key words: ATR, gemcitabine, combination treatment, DNA damage, pancreatic cancer cooperative induction of cell death in the pancreatic cancer cell lines. AZ20 significantly increased GEM-induced DNA damage and almost completely abrogated GEM-induced expression of the M2 subunit of ribonucleotide reductase. These findings suggest that inhibition of ATR is a promising strategy to enhance the antitumor activity of GEM for treating pancreatic cancer.

\section{Introduction}

Pancreatic cancer is a disease with high mortality due to the lack of early detection and resistance to gemcitabine (GEM)-based chemotherapy. More than $90 \%$ of pancreatic cancer cases are found to be metastatic at diagnosis. In addition, over $80 \%$ of patients newly diagnosed with this disease are not eligible for surgical resection (1). These factors lead to an extremely low 5-year survival rate of patients with pancreatic cancer, which is only $7.2 \%$ (2). Drug intervention is the general treatment for pancreatic cancer. GEM has been used as the standard chemotherapeutic agent for pancreatic cancer since 1997. However, the clinical efficacy of GEM monotherapy is very limited mainly due to drug resistance. Therefore, developing new rational combination treatments with GEM is a promising strategy to overcome GEM resistance in pancreatic cancer (3-5).

Once inside the cell, GEM is phosphorylated to its monophosphate (dFdCMP), diphosphate (dFdCDP) and triphosphate (dFdCTP) form consecutively (4). dFdCTP incorporates into replicating DNA strands, resulting in termination of DNA synthesis, induction of DNA replication stress and DNA damage, and activation of the DNA damage response (DDR), leading to GEM resistance (6). In addition, dFdCDP also inhibits ribonucleotide reductase (RR), and consequently decreased NTP pools (4). RR is composed of the regulatory subunit (RRM1) and catalytic subunit (RRM2). Both RRM1 and RRM2 overexpression is associated with GEM 
resistance $(7,8)$. Thus, combination of GEM with a drug which targets the DDR and RR may enhance its antitumor activity against pancreatic cancer.

Ataxia telangiectasia mutated and Rad3-related (ATR), a key regulator of the DDR, has important functions in sensing and repairing DNA damage, regulating the cell cycle, stabilizing replication forks and restraining replication origin firing (9-11). Furthermore, a recent study suggests that ATR coordinates RRM2 accumulation to suppress replication catastrophe (12). Thus, targeting ATR could potentially overcome GEM resistance by inhibiting DNA repair, abrogating GEM-induced activation of the cell cycle checkpoints, and suppressing RR expression.

Cancer cells frequently harbor deficiencies in one or more DDR pathways. These DDR deficiencies not only contribute to tumorigenesis, but also render the cancer cells more dependent on the remaining functional DDR pathways. Thus, targeting ATR to treat pancreatic cancer may not only enhance GEM sensitivity, but also show tumor selectivity (13-15). Thus, ATR has been regarded as a promising target for cancer treatment. Growing evidence shows that ATR inhibition increases the antitumor activity of chemotherapeutic agents including GEM and radiation in a variety of cancers including pancreatic cancer $(16,17)$. Inhibition of ATR sensitizes pancreatic cancer cells to radiation accompanied by inhibition of homologous recombination repair, decrease of checkpoint activation and induction of more DNA damage $(18,19)$. However, the underlying molecular mechanisms are not fully understood.

In the present study, we chose a novel ATR-selective inhibitor, AZ20, which has shown potent anti-colorectal tumor activity in both in vitro and in vivo preclinical models (20), to investigate the antitumor effect and the underlying molecular mechanism of ATR inhibition either alone or in combination with GEM in pancreatic cancer cell lines. Our results showed that although AZ20 treatment had limited effect on cell death, it significantly enhanced that induced by GEM. Notably, AZ20 enhanced GEM-induced cell death mainly through enhancement of GEM-induced DNA damage rather than abrogation of cell cycle checkpoints. It has been hypothesized that p53-deficient cancer cells (resulting in loss of G1 checkpoint) rely on the ATR/CHK1 pathway for DNA damage repair and are more sensitive to ATR inhibition (21-24). Our data suggests that the antitumor activity of the combination of AZ20 and GEM was independent of the p53 status. These findings suggest that targeting ATR may represent a promising strategy to overcome GEM resistance in pancreatic cancer.

\section{Materials and methods}

Drugs. AZ20, GEM and roscovitine were purchased from Selleck Chemicals (Houston, TX, USA).

Cell lines and treatments. The AsPC-1, BxPC-3, CFPAC-1, HPAC and MIAPaCa-2 human pancreatic cancer cell lines were purchased from the American Type Culture Collection (ATCC; Manassas, VA, USA), and were authenticated by the University of Arizona Genetics Core Facility (Tucson, AZ, USA). The cells were cultured in Dulbecco's modified Eagle's medium (DMEM; Invitrogen, Carlsbad, CA, USA; HPAC and MIAPaCa-2), RPMI-1640 medium (Invitrogen; AsPC-1 and
BxPC-3) or Iscove's modified Dulbecco's medium (IMDM; Invitrogen; CFPAC-1) with 10\% heat-inactivated fetal bovine serum (FBS; HyClone Laboratories, Logan, UT, USA) plus $100 \mathrm{U} / \mathrm{ml}$ penicillin and $100 \mu \mathrm{g} / \mathrm{ml}$ streptomycin in a $37^{\circ} \mathrm{C}$ humidified atmosphere containing $5 \% \mathrm{CO}_{2} / 95 \%$ air. The cell lines were tested for the presence of mycoplasma on a monthly basis.

In vitro cytotoxicity assays. In vitro cytotoxicity of AZ20 and GEM, alone or in combination, was determined using 3-[4,5-dimethylthiazol-2-yl]-2,5-diphenyltetrazolium bromide (MTT) (Sigma-Aldrich, St. Louis, MO, USA) reagent, as previously described (25). $\mathrm{IC}_{50}$ values were calculated as drug concentrations necessary to inhibit $50 \%$ cell growth compared to vehicle control-treated cells using GraphPad Prism 5.0 software (GraphPad Software, San Diego, CA, USA). The extent and direction of antitumor interactions between AZ20 and GEM were determined by calculating combination index (CI) values. $\mathrm{CI}<0.9$ indicates synergistic, $0.9<\mathrm{CI}<1.1$ indicates additive, and $\mathrm{CI}>1.1$ indicates antagonistic antitumor interactions, respectively.

Cell death and cell cycle progression. Pancreatic cancer cell lines were treated with the indicated drugs for up to $48 \mathrm{~h}$. Cells were fixed with ice-cold $80 \%$ (v/v) ethanol for $24 \mathrm{~h}$ at $4^{\circ} \mathrm{C}$. The cells were pelleted, then were washed with phosphate-buffered saline (PBS) ( $\mathrm{pH}$ 7.4) and resuspended in PBS containing propidium iodide (PI; $50 \mu \mathrm{g} / \mathrm{ml})$, Triton X-100 $(0.1 \%$, v/v), and DNase-free RNase $(1 \mu \mathrm{g} / \mathrm{ml})$. DNA content was determined by flow cytometric analysis using a FACSCalibur flow cytometer (Becton-Dickinson, San Jose, CA, USA), as previously described (25). Cell death events were expressed as the percentage of cells with sub-G1 DNA content. Cell cycle analysis and histogram generation were carried out using FlowJo v7.6.5 (Tree Star, Ashland, OR, USA).

Western blot analysis. Soluble proteins were extracted in the presence of protease and phosphatase inhibitors (Roche Applied Sciences China Inc., Shanghai, China) and subjected to SDS-polyacrylamide gel electrophoresis. Separated proteins were electrophoretically transferred onto polyvinylidene difluoride (PVDF) membranes (Thermo Fisher Inc., Rockford, IL, USA) and immunoblotted with anti-PARP-1 (9542), -pCDK1 (Y15) (9111), -CDK2 (2546), - $\gamma$ H2AX (2577), -GAPDH (2118; Cell Signaling Technology, Danvers, MA, USA), -CHK1 (sc8408, Santa Cruz Biotechnology, Santa Cruz, CA, USA), -RRM1 (ab137114), -RRM2 (ab172476), -pCHK1 (S345) (ab47318), -pCDC25C (S216) (ab32051), -pCDK2 (Y15) (ab76146) or -CDK1 (ab32094; Abcam, Cambridge, MA, USA) antibodies, as previously described (25). Primary antibodies were diluted 1:1,000 in Odyssey Blocking Buffer (Li-Cor, Lincoln, NE, USA). Immunoreactive proteins were visualized using the Odyssey Infrared Imaging System (Li-Cor). Western blot analyses were repeated 3 times and one representative blot is shown.

Alkaline comet assay. BxPC-3 or HPAC cells were treated with the indicated drugs for $8 \mathrm{~h}$, and then subjected to alkaline comet assay as previously described (26). Slides were stained with SYBR Gold (Invitrogen), and then imaged on an Olympus 
A<smiles>CC1COCCN1c1cc(C2(S(C)(=O)=O)CC2)nc(-c2cccc3[nH]ccc23)n1</smiles>

AZ20

C

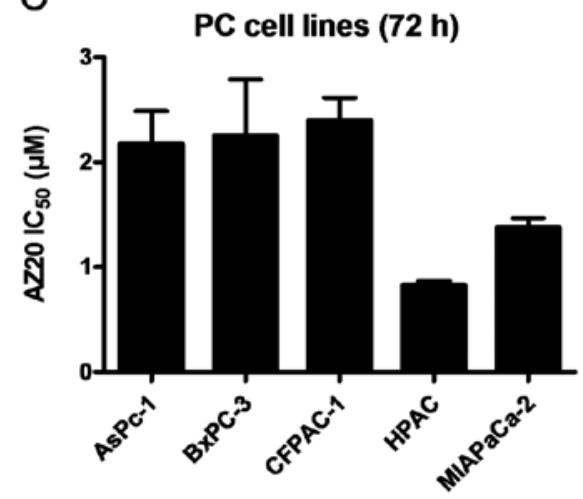

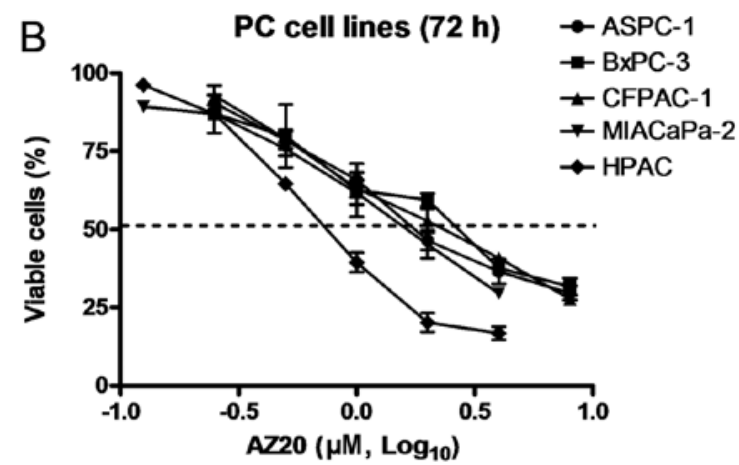

D

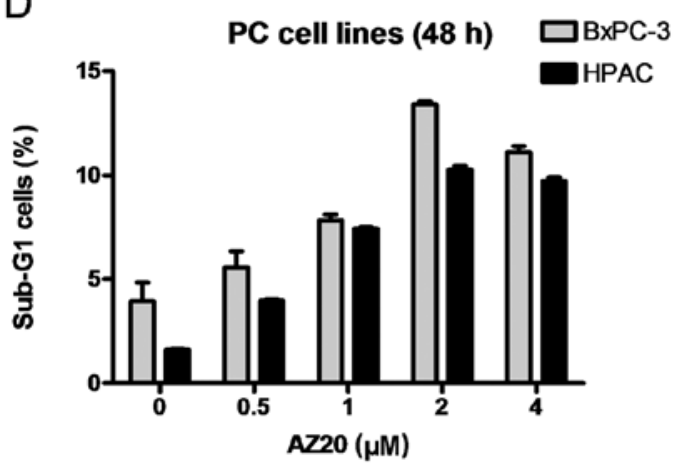

Figure 1. AZ20 impairs cell viability and induces limited cell death in pancreatic cancer cell lines. (A) The chemical structure of AZ20, a novel ATR-selective inhibitor. (B and C) Pancreatic cancer cell lines were cultured in 96-well plates with variable concentrations of AZ20 for $72 \mathrm{~h}$ and viable cells were determined

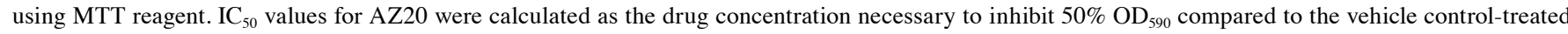
cells. The data are presented as means \pm standard errors from at least 3 independent experiments. (D) BxPC-3 and HPAC cells were treated with variable concentrations of AZ20 for $48 \mathrm{~h}$. The cells were fixed with $80 \%$ ethanol, stained with PI and subjected to flow cytometric analysis. Dead cells are expressed as the percentage of $\mathrm{PI}^{+}$cells with sub-G1 DNA content. The data are presented as means of triplicates \pm standard errors from one representative experiment.

IX73 microscope equipped with a DP80 digital camera for microscope and cellSens EN-V1 software (Olympus China Inc., Beijing, China). At least 100 comets/gel were scored by CometScore (TriTek Corp., Sumerduck, VA, USA). The median percentage of DNA in the tail was calculated and expressed as mean \pm SEM.

Statistical analysis. Differences were compared using the pair-wise two-sample t-test. Statistical analyses were performed using GraphPad Prism 5.0. Error bars represent \pm SEM. The level of significance was set at $\mathrm{p}<0.05$.

\section{Results}

AZ20 treatment causes growth arrest, but limited cell death in pancreatic cancer cells. To begin our investigation, the sensitivity of AZ20 was determined in a panel of pancreatic cancer cell lines using MTT assays after $72 \mathrm{~h}$ of drug treatment. As shown in Fig. 1B and C, AZ20 treatment caused growth inhibition in a concentration-dependent manner with $\mathrm{IC}_{50}$ values ranging from $0.8 \mu \mathrm{M}$ in HPAC cells to $2.4 \mu \mathrm{M}$ in CFPAC-1 cells. In contrast to a previous hypothesis that p53-deficient cancer cells are more sensitive to ATR inhibition, our data showed that p53-wild-type HPAC cells responded better to AZ20 treatment compared to the p53-mutant pancreatic cancer cell lines (Fig. 1C). Since HPAC and BxPC-3 cell lines are widely used in preclinical studies, we chose these 2 cell lines for the rest of our studies.
To determine whether AZ20 treatment causes cell death, BxPC-3 and HPAC cells were treated with variable concentrations of AZ20 for $48 \mathrm{~h}$ and then subjected to flow cytometric analysis to detect sub-G1 cells. As compared to the no drug treatment control, AZ20 treatment induced statistically significant but biologically limited cell death $(<14 \%)$. This was accompanied by low levels of PARP-1 cleavage in both cell lines, indicating that the AZ20 treatment-induced cell death was through apoptosis (Figs. 1D, and 2C and D). These results demonstrated that AZ20 treatment causes growth inhibition and limited cell death in the pancreatic cancer cells.

AZ20 treatment induces cell cycle arrest and DNA damage in pancreatic cancer cells. To investigate the effect of AZ20 on cell cycle progression in pancreatic cancer cells, we treated BxPC-3 and HPAC cells with the indicated concentrations of AZ20, and then analyzed cell cycle distribution in the cells by PI staining and flow cytometric analyses. AZ20 treatment induced $\mathrm{S}$ and $\mathrm{G} 2 / \mathrm{M}$ arrest in a dose-dependent manner in the BxPC-3 cells (Fig. 2A). Similar results were also obtained in the HPAC cells (Fig. 2B). These results showed that the effect of AZ20 treatment on cell cycle checkpoints is activation rather than abrogation, suggesting that AZ20 treatment caused DNA damage in the cells.

To test this possibility, we investigated the effects of AZ20 treatment on the expression of $\gamma \mathrm{H} 2 \mathrm{AX}$, a biomarker of DNA double-strand breaks (27), and the downstream signaling of ATR. In both BxPC-3 and HPAC cells, AZ20 treatment 
A

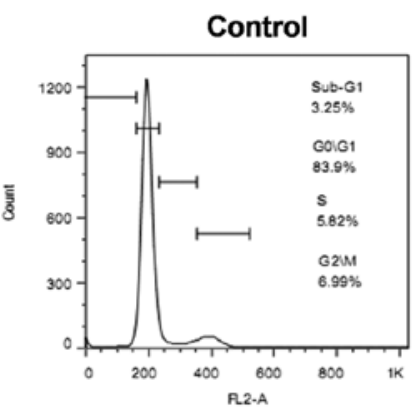

$2 \mu \mathrm{M}$

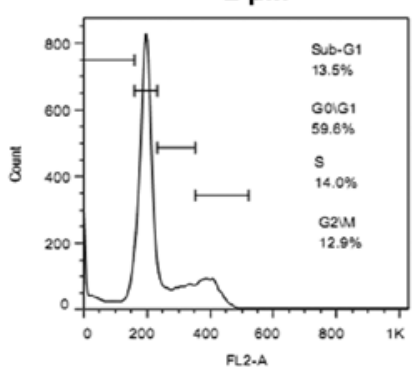

B

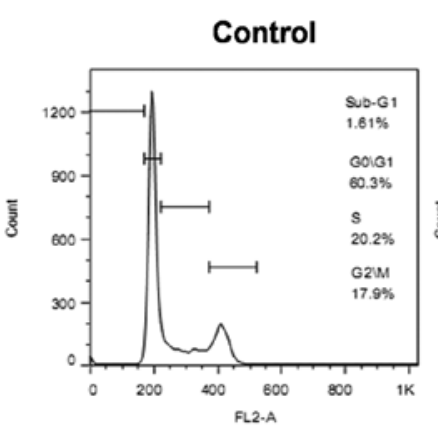

$2 \mu \mathrm{M}$

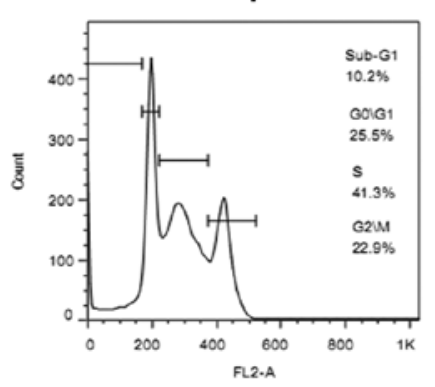

BxPC-3 (48 h)

$0.5 \mu \mathrm{M}$

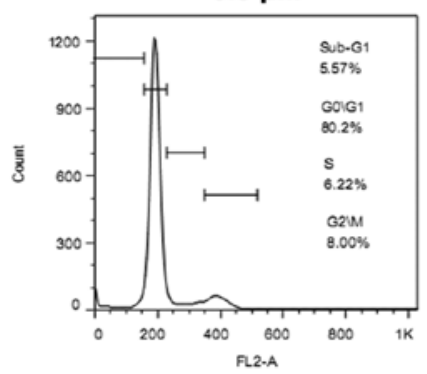

$4 \mu \mathrm{M}$

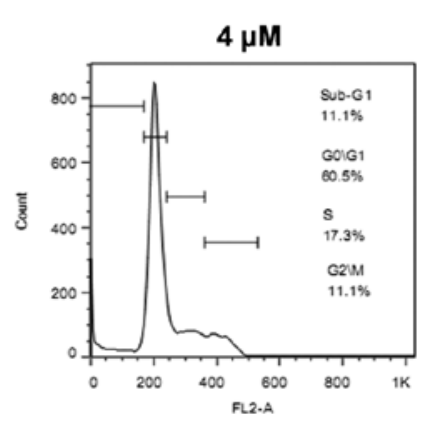

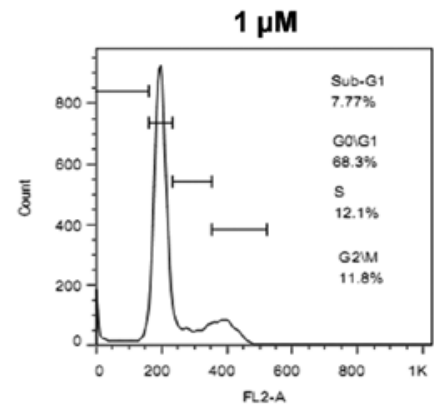

\section{HPAC (48 h)}
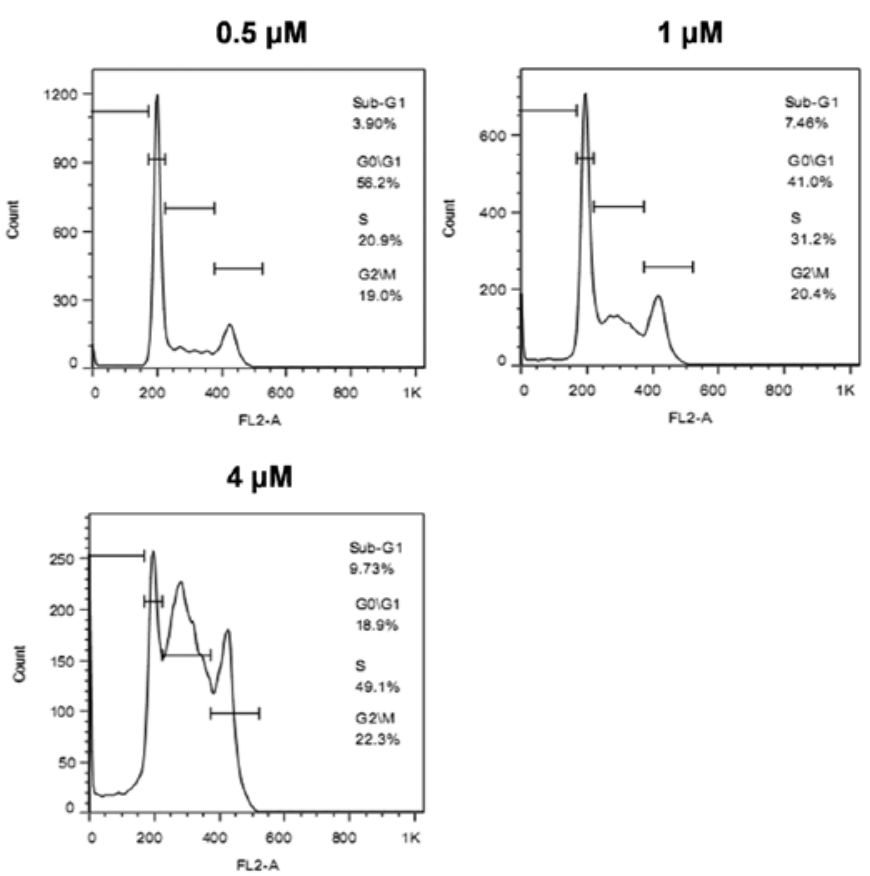

Figure 2. AZ20 induces cell cycle arrest, inhibits the activity of CHK1, and increases DNA damage in pancreatic cancer cells. (A and B) BxPC-3 and HPAC cells were treated with variable concentrations of AZ20 for $48 \mathrm{~h}$. The cells were fixed with $80 \%$ ethanol, stained with PI, and subjected to flow cytometric analysis to determine cell cycle distribution. Representative histograms are shown.

increased the expression of $\gamma \mathrm{H} 2 \mathrm{AX}$, indicative of DNA damage. AZ20 also caused obviously decreased phosphorylation of CHK1 at serine-345, CDC25C at serine-216 and CDK1 at tyrosine-15, demonstrating suppression of the CHK1/ CDC25C/CDK1 pathway resulting from ATR inhibition. In contrast, AZ20 treatment increased the phosphorylation of CDK2 at tyrosine-15 (Fig. 2C and D). It has recently been reported that inhibition of ATR causes decreased expression of RRM2 in cancer cells (12). Although AZ20 treatment caused downregulation of RRM2 in BxPC-3 cells, it had no effect on RRM2 expression in HPAC cells. Notably, AZ20 treatment substantially decreased the expression of RRM1 in the BxPC-3 and in HPAC cells, although to a much lesser extent (Fig. $2 \mathrm{C}$ and $\mathrm{D}$ ). These data suggest that the induction of DNA damage by AZ20 was partially due to its negative effect on RR expression. 

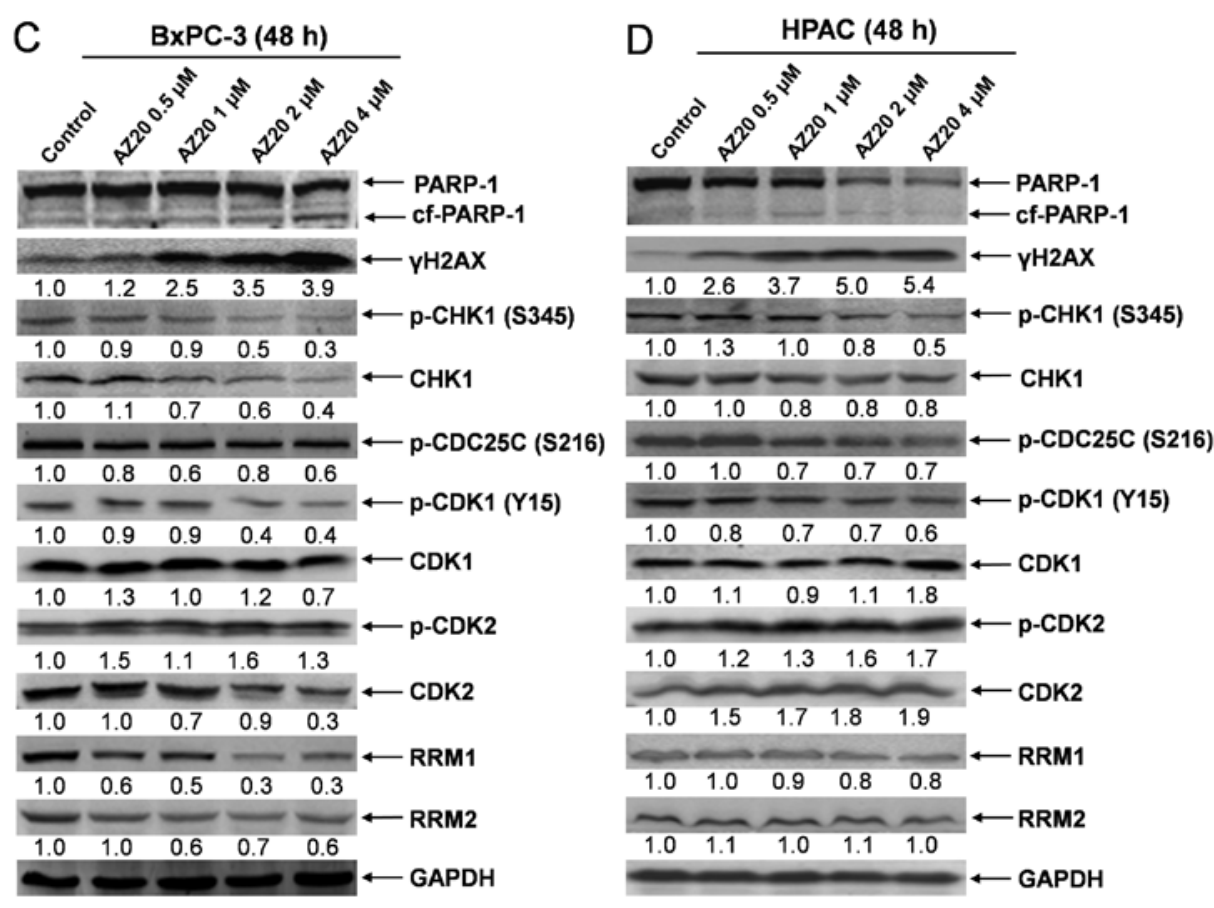

Figure 2. Continued. (C and D) BxPC-3 and HPAC cells were treated with variable concentrations of AZ20 for 48 h. Whole cell lysates were subjected to western blotting and probed with anti-PARP-1, $\gamma \mathrm{H} 2 \mathrm{AX}$, -p-CHK1, -CHK1, -p-CDC25C, -p-CDK1, -CDK1, -p-CDK2, -CDK2, -RRM1, -RRM2 or -GAPDH antibody.

AZ20 enhances GEM-induced DNA damage and cell death. To examine the effect of AZ20 on GEM-induced cell death and cell cycle progression, BxPC-3 and HPAC cells were treated with $1 \mu \mathrm{M}$ AZ20 and $80 \mathrm{nM}$ GEM alone or in combination for $48 \mathrm{~h}$. Compared to the single drug treatment, AZ20 and GEM combination caused significantly increased cell death accompanied by substantially increased PARP-1 cleavage and $\gamma \mathrm{H} 2 \mathrm{AX}$ expression in both cell lines (Fig. 3A, F and G). The presence of AZ20 also significantly decreased GEM-induced $\mathrm{S}$ phase arrest in both cell lines, and $\mathrm{G} 2 / \mathrm{M}$ phase arrest in BxPC-3 cells (Fig. 3B-E). AZ20 suppressed GEM-induced CHK1 phosphorylation at serine-345, indicating a negative effect on GEM-induced checkpoint activation. Although GEM treatment had differential effects on the phosphorylation of CDC25C (serine-216) in BxPC-3 and HPAC cells (induction in BxPC-3 cells, while suppression in HPAC cells), it increased tyrosine-15 phosphorylation of CDK1/2 in both cell lines, which was almost completely abolished by AZ20 (Fig. 3F and G). These data indicate that AZ20 increases GEM-induced cell death through abrogation of cell cycle checkpoints and enhancement of DNA damage.

Consistent with previous studies $(7,8)$, GEM treatment resulted in increased protein levels of RRM1 in the HPAC cells and RRM 2 in both BxPC-3 and HPAC cell lines. Notably, addition of AZ20 almost completely abolished GEM-induced RR expression in both cell lines (Fig. 3F-H). These results suggest that AZ20 enhances GEM sensitivity in pancreatic cancer cells, at least partially through abrogation of GEM-induced RR expression.

AZ20 increases GEM-induced DNA damage at an early time point. Previous studies have demonstrated that DNA fragmentation at the late stage of cell apoptosis also induces $\gamma \mathrm{H} 2 \mathrm{AX}$ expression (27). To provide direct evidence that the AZ20 and GEM combination indeed cooperate in inducing DNA damage, we determined the expression of $\gamma \mathrm{H} 2 \mathrm{AX}$ and other relevant proteins in BxPC-3 and HPAC pancreatic cancer cells post $8 \mathrm{~h}$ drug treatments. Consistent with the results obtained in the cells post $48 \mathrm{~h}$ drug treatments, the combination treatment resulted in substantially increased expression of $\gamma \mathrm{H} 2 \mathrm{AX}$ in the cells compared to individual drug treatment. Neither single nor combination drug treatment had obvious effects on cell morphology and PARP-1 cleavage, demonstrating that the drug treatments did not induce cell death under these conditions. AZ20 treatment also abolished GEM-induced RRM2 expression in the cells. In contrast, there was no obvious effect of the drug treatments on the CHK1/CDC25C/CDK1/2 pathway proteins (Fig. 4A-C). These results suggest that AZ20 enhances the antitumor activity of GEM mainly by increasing GEM-induced DNA damage and decreasing GEM-induced RRM2 expression rather than abrogating GEM-induced checkpoint activation.

To further confirm the effect of AZ20 on GEM-induced DNA damage, comet assay was performed after $8 \mathrm{~h}$ drug treatment. As shown in Fig. 4D-G, both AZ20 and GEM induced DNA strand breaks, as assessed by the percentage of DNA content in the comet tail compared to the no drug treatment control. Furthermore, combined treatment with AZ20 with GEM significantly increased DNA strand breaks compared to the single drug treatment in both BxPC-3 and HPAC cell lines. These results provide compelling evidence that AZ20 enhances GEM-induced DNA damage prior to cell death in pancreatic cancer cells.

AZ20 increases the antitumor activity of GEM in a synergistic manner. Finally, we determined the direction and extent 

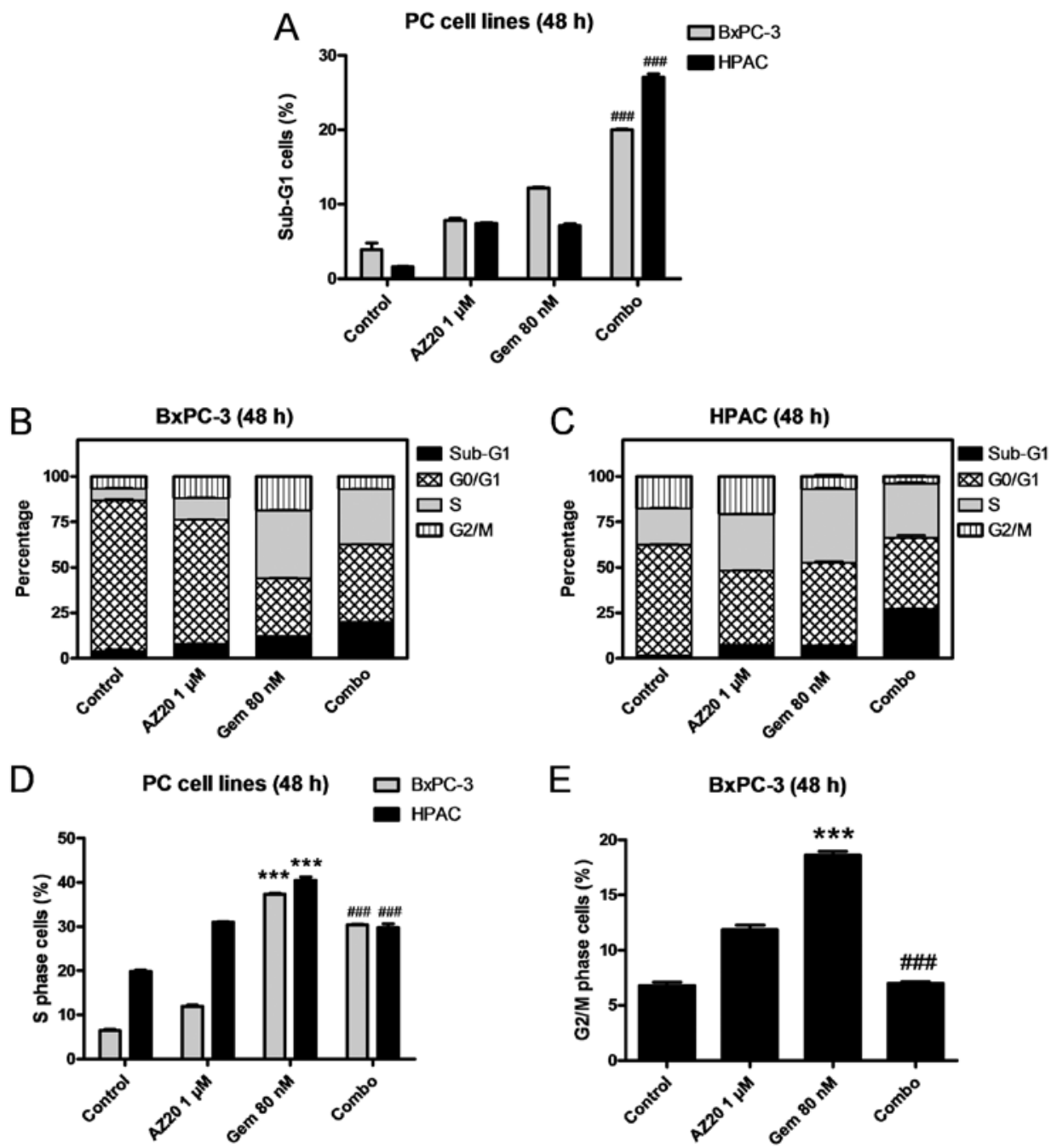

E
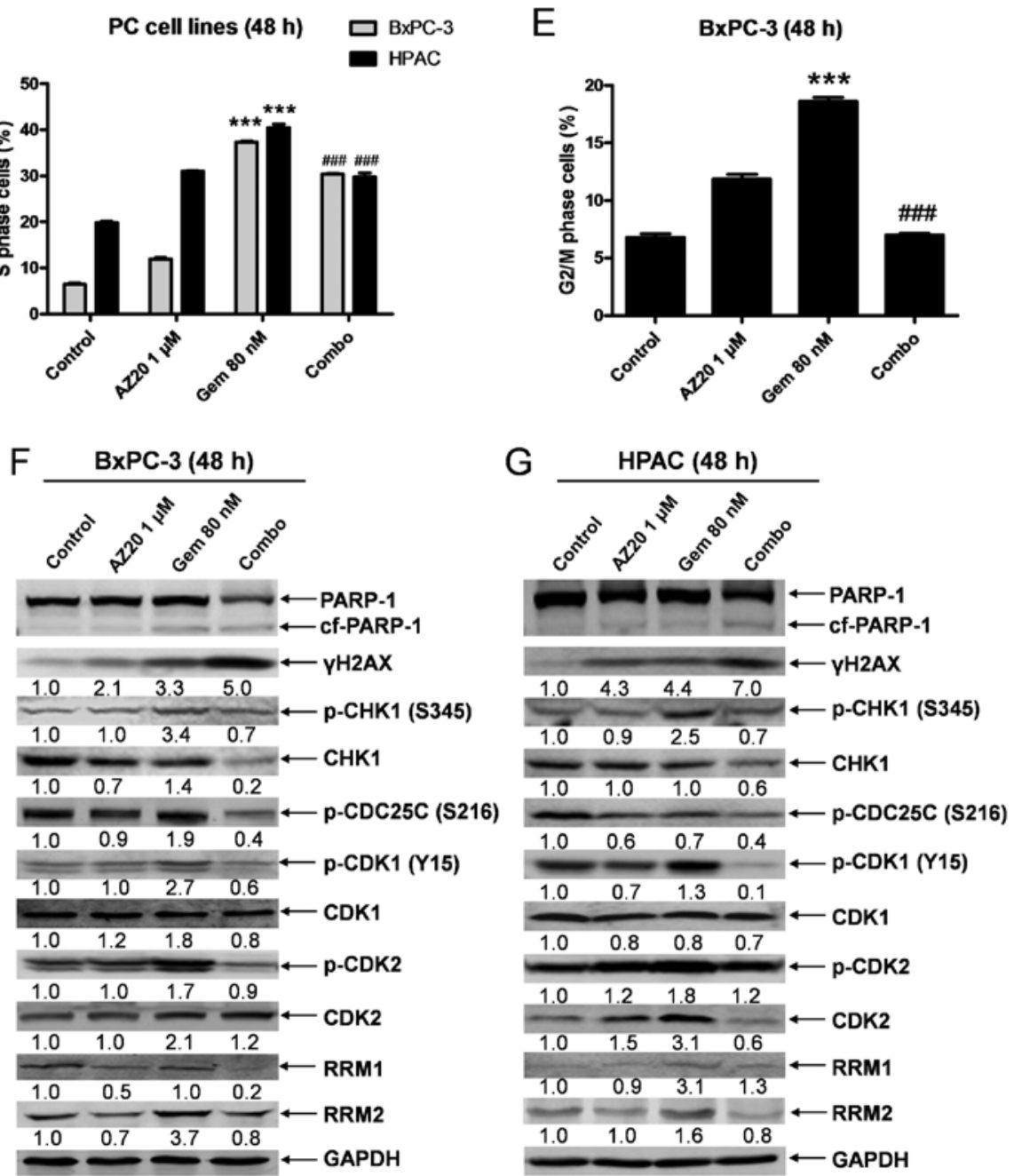

Figure 3. AZ20 enhances GEM-induced cell death in pancreatic cancer cells. (A) BxPC-3 and HPAC cells were treated with $1 \mu$ M AZ20 and $80 \mathrm{nM}$ GEM alone or simultaneously for $48 \mathrm{~h}$. The cells were fixed with $80 \%$ ethanol, stained with PI and subjected to flow cytometric analysis to determine cell death (sub-G1) and cell cycle distribution. The data are presented as means of triplicates \pm standard errors from 1 representative experiment. (B-E) Cell cycle results particularly the percentage of cells in the S phase in BxPC-3 and HPAC cell lines and G2/M phase in the BxPC-3 cells are graphed as means of triplicates \pm standard errors from 1 representative experiment. ( $\mathrm{F}$ and G) BxPC-3 and HPAC cells were treated with $1 \mu \mathrm{M}$ AZ20 and $80 \mathrm{nM}$ GEM alone or simultaneously for $48 \mathrm{~h}$. Whole cell lysates were subjected to western blotting and probed with anti-PARP-1, - $\gamma \mathrm{H} 2 \mathrm{AX},-\mathrm{p}-\mathrm{CHK} 1,-\mathrm{CHK} 1,-\mathrm{p}-\mathrm{CDC} 25 \mathrm{C}$, -p-CDK1, -CDK1, -p-CDK2, -CDK2, -RRM1, -RRM2 or -GAPDH antibody; ${ }^{*}$ p $<0.05$ (GEM vs. control), ${ }^{* *}$ p $<0.005$ (GEM vs. control), ${ }^{* * * *}$ p $<0.001$ (GEM vs. control), ${ }^{\# \#} \mathrm{p}<0.005$ (combo vs. GEM), ${ }^{\# \#} \mathrm{p}<0.001$ (combo vs. GEM). 


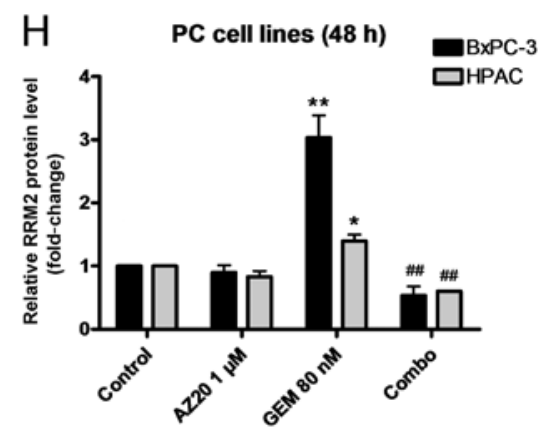

Figure 3. Continued. (H) Relative RRM2 protein levels are expressed as means \pm standard errors from 3 independent experiments; "p $<0.05$ (GEM vs. control), ${ }^{* *} \mathrm{p}<0.005$ (GEM vs. control), ${ }^{* * *} \mathrm{p}<0.001$ (GEM vs. control), ${ }^{\# \#} \mathrm{p}<0.005$ (combo vs. GEM), ${ }^{\# \# \#} \mathrm{p}<0.001$ (combo vs. GEM).

Table I. AZ20 enhances GEM sensitivity in a synergistic manner in pancreatic cancer cell lines.

\begin{tabular}{lcccccc}
\hline & & \multicolumn{2}{c}{$\mathrm{IC}_{50}$ of gemcitabine $(\mathrm{nM})$ in the absence or presence of $\mathrm{AZ20}(\mu \mathrm{M})$} & \\
\cline { 3 - 6 } Cell line & $\mathrm{IC}_{50}$ of AZ20 $(\mu \mathrm{M})$ & 0 & 0.25 & 0.5 & 1 & \multirow{2}{*}{ aP-value } \\
\hline ASPC-1 & $2.4 \pm 0.3$ & $657.5 \pm 59.7$ & $255.8 \pm 29.3(0.49)$ & $141.0 \pm 23.3(0.42)$ & $109.6 \pm 27.7(0.58)$ & $<0.001$ \\
BxPC-3 & $2.3 \pm 0.5$ & $26.6 \pm 5.0$ & $13.7 \pm 2.0(0.63)$ & $8.6 \pm 0.7(0.55)$ & $4.6 \pm 0.2(0.62)$ & $<0.05$ \\
CFPAC-1 & $2.4 \pm 0.2$ & $6.5 \pm 0.8$ & $3.8 \pm 0.5(0.69)$ & $2.7 \pm 0.2(0.62)$ & $2.0 \pm 0.3(0.72)$ & $<0.05$ \\
HPAC & $0.8 \pm 0.04$ & $14.7 \pm 1.9$ & $3.8 \pm 0.3(0.56)$ & $1.9 \pm 0.1(0.73)$ & ND & $<0.005$ \\
MIAPaCa-2 & $1.4 \pm 0.09$ & $14.7 \pm 1.7$ & $5.1 \pm 0.5(0.52)$ & $3.3 \pm 0.4(0.59)$ & $2.6 \pm 0.5(0.90)$ & $<0.005$ \\
\hline
\end{tabular}

The $\mathrm{IC}_{50}$ values of AZ20 and GEM are presented as means \pm standard errors from 3 independent experiments. Numbers in the parentheses represent the combination index values. $\mathrm{CI}<0.9$, synergistic, $0.9<\mathrm{CI}<1.1$, additive and $\mathrm{CI}>1.1$, antagonistic antitumor interactions. $\mathrm{ND}$, not determined. ${ }^{\mathrm{a}} \mathrm{P}$-values for each pair were determined using GraphPad Prism 5.0 and only the largest p-value is shown for each cell line. GEM, gemcitabine; CI, combination index.

of antitumor interactions between AZ20 and GEM in the 5 pancreatic cancer cells by MTT assays and calculating CI values using CalcuSyn software. As shown in Table I, addition of AZ20 significantly decreased the $\mathrm{IC}_{50}$ values of GEM in each of the pancreatic cancer cell lines tested. By calculating CI values using the CalcuSyn software, we demonstrated that the antitumor interactions between the 2 agents were synergistic $(\mathrm{CI}<0.9)$. These results demonstrated that combination of GEM with AZ20 had synergistic antitumor activities in pancreatic cancer cells.

\section{Discussion}

Previous studies have mainly focused on the role of ATR in cell cycle checkpoints, and suggest that abrogation of cell cycle checkpoints is the main mechanism by which ATR inhibitors synergize with DNA damaging agents $(18,19)$. In contrast to these previous studies, in the present study we found that the novel ATR inhibitor AZ20 enhanced the antitumor activity of GEM mainly by enhancing GEM-induced DNA double-strand breaks and by abolishing GEM-induced RRM2 expression rather than abrogating GEM-induced checkpoint activation.

Similar to studies using DNA damaging agents in combination with ATR inhibitors $(18,19,28)$, we found that AZ20 synergized with GEM to induce apoptosis in pancreatic cancer cells. AZ20 abrogated GEM-induced p-CHK1 and RRM2 expression, while enhancing GEM-induced DNA damage. Although we found that AZ20 treatment reduced the percentages of GEM-induced $\mathrm{S}$ and G2/M phase cells, the amount of reduction was not very substantial at $48 \mathrm{~h}$ post-treatment. At
$8 \mathrm{~h}$ post combined AZ20 and GEM treatment, we did not detect changes in CDK1/2 phosphorylation, although we did detect a significant increase in DNA damage. Based on these results, we believe that the effects of AZ20 on cell cycle distribution were due to death of cells in the $\mathrm{S}$ and $\mathrm{G} 2 / \mathrm{M}$ phases rather than abrogation of the cell cycle checkpoints. Fokas et al used the selective ATR inhibitor VE-822 in pancreatic cancer cells and found that it caused G1 arrest, although they did not determine levels of phosphorylated CDK1 and CDK2 (19). However, they found that VE-822 had no effect on GEM-induced S phase arrest (19). Their results are similar to our results, further supporting our findings that ATR inhibition enhances GEM through means other than cell cycle checkpoint abrogation.

ATR inhibitors were developed based on the evidence that ATR mediates $\mathrm{S}$ and G2/M cell cycle arrest. Thus, in cells with deficient G1 checkpoints, DNA damage repair may rely on the $S$ and $\mathrm{G} 2 / \mathrm{M}$ checkpoints for repair. Inhibition of ATR in G1-deficient cells may result in accumulation of DNA damage and cause cell death $(17,29)$. Several studies have since supported this hypothesis and have demonstrated that ATR inhibitors abrogate the S and G2/M cell cycle checkpoints (reviewed in ref. 17). However, in the present study we used p53 wild-type HPAC cells and found that the ATR inhibitor AZ20 enhanced GEM-induced cell death. Our finding suggests that AZ20 did not enhance GEM by abrogating the $S$ and $\mathrm{G} 2 / \mathrm{M}$ cell cycle checkpoints rather that AZ20 enhanced GEM-induced DNA damage in pancreatic cancer cells.

ATR inhibition has been demonstrated to cause downregulation of RRM2 (12,30). Consistent with that study, our results showed increased RRM2 after treatment with GEM, 

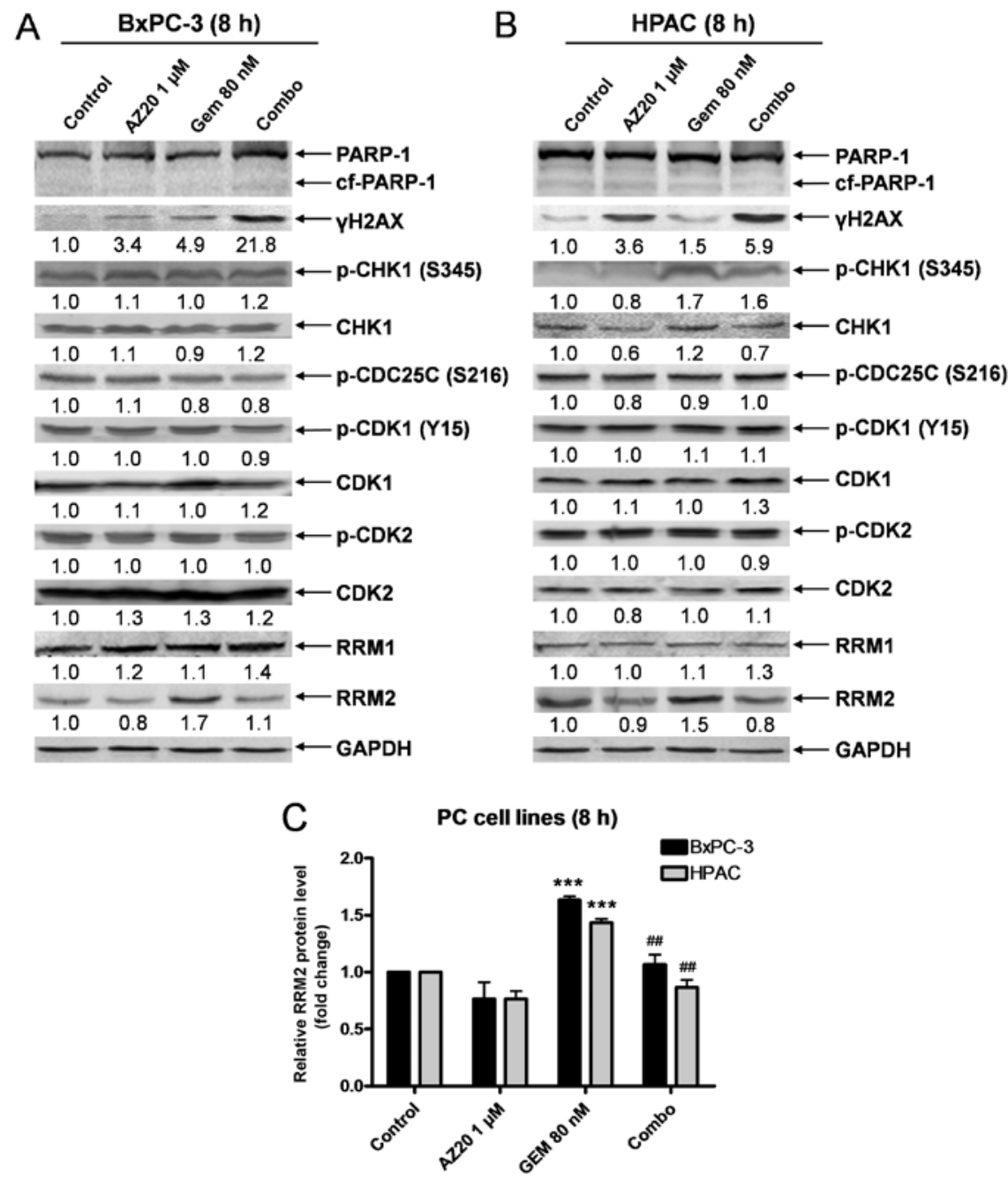

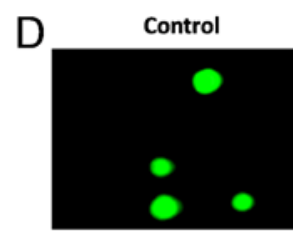

Gem $80 \mathrm{nM}$
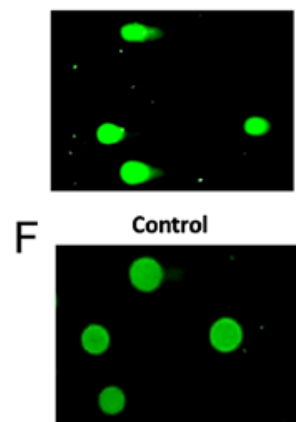

Gem 80 nM

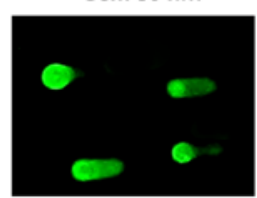

AZ20 $1 \mu \mathrm{M}$

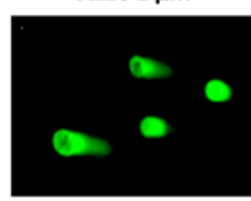

Combo

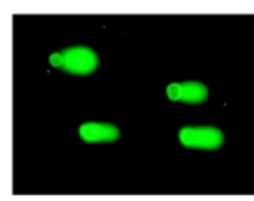

AZ20 $1 \mu \mathrm{M}$

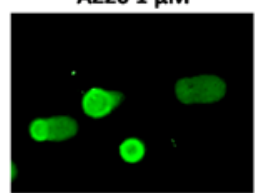

Combo

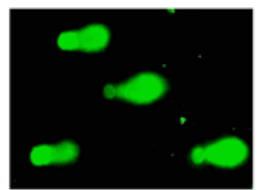

$\mathrm{E}$

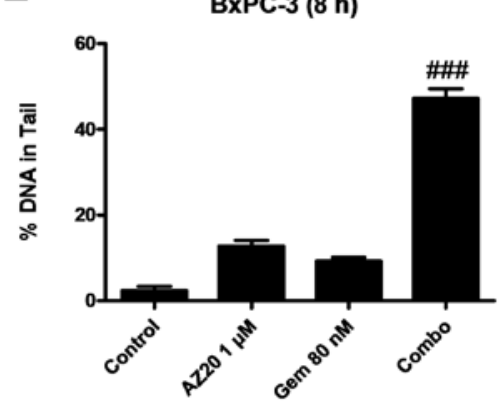

G

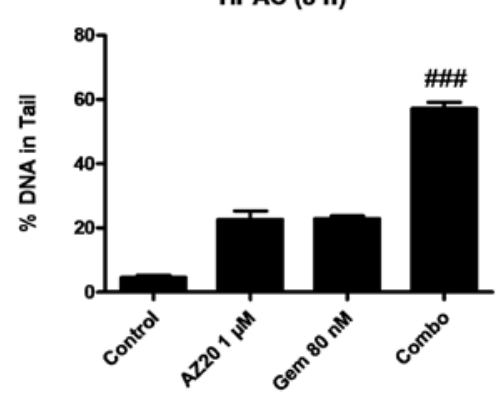

Figure 4. AZ20 enhances GEM-induced DNA damage in pancreatic cancer cells. (A and B) BxPC-3 and HPAC cells were treated with $1 \mu$ M AZ20 and $80 \mathrm{nM}$ GEM alone or simultaneously for $8 \mathrm{~h}$. Whole cell lysates were subjected to western blotting and probed with anti-PARP-1, $-\gamma \mathrm{H} 2 \mathrm{AX},-\mathrm{p}-\mathrm{CHK} 1,-\mathrm{CHK} 1$, -p-CDC25C, -p-CDK1, -CDK1, -p-CDK2, -CDK2, -RRM1, -RRM2 or -GAPDH antibody. (C) Relative RRM2 protein levels are graphed as means \pm standard errors from 3 independent experiments. (D and E) BxPC-3 and HPAC cells were treated with $1 \mu \mathrm{M}$ AZ20 and $80 \mathrm{nM}$ GEM alone or simultaneously for $8 \mathrm{~h}$, and then the cells were subjected to alkaline comet assay. Representative comets are shown. (F and G) Comet assay results are graphed as the median percentage of DNA in the tail from 3 replicate gels \pm SEM; ${ }^{* * *}$ p $<0.001$ (GEM vs. control), ${ }^{\# \#}$ p $<0.005$ (combo vs. GEM), ${ }^{\# \# \#<0.001 ~(c o m b o ~ v s . ~ G E M) . ~}$ 
which was decreased by the addition of AZ20. Moreover, GEM-induced DNA damage was significantly enhanced by AZ20. In addition to RRM2, RRM1 modulation has been shown to directly influence GEM efficacy (31). Zhou et al performed a kinome screen to identify sensitizers for RRM1-dependent GEM efficacy (32). They identified CHK1 as a major therapeutic target capable of overcoming GEM resistance in non-small cell lung cancer. Our results are similar in that targeting ATR, directly upstream of CHK1, enhanced GEM-induced apoptosis in pancreatic cancer cells. In BxPC-3 cells, GEM treatment alone caused downregulation of RRM1, which was further downregulated when combined with GEM (48 $\mathrm{h}$ treatment). While in HPAC cells, AZ20 treatment alone did not have an effect on RRM1 and RRM2, though it did abrogate induction of RRM1 and RRM2 by GEM treatment. It has been reported that knockdown of RRM2 could lead to apoptosis in cancer cells (33). Thus, abrogation of GEM-induced RRM2 expression by AZ20 may enhance GEM-induced cell death in pancreatic cancer cells. However, changes in RRM2 were detected after treatment for only $8 \mathrm{~h}$, while the effects on RRM1 were not detected until later, suggesting that the effects on RRM2 contributed to the enhanced cell killing, whereas contribution from RRM1 may have been consequential rather than causal.

Taken together, our data showed that targeting ATR using AZ20 can enhance the antitumor activity of GEM through induction of DNA damage and decrease in RRM2 expression in pancreatic cancer cells. Accordingly, combination of AZ20 with GEM may represent a potential chemotherapeutic regimen for treating pancreatic cancer. Although our studies were limited to in vitro models, our findings support the further development of AZ20 in combination with GEM for the treatment of pancreatic cancer.

\section{References}

1. Cid-Arregui A and Juarez V: Perspectives in the treatment of pancreatic adenocarcinoma. World J Gastroenterol 21 : 9297-9316, 2015.

2. Majumder S, Chari ST and Ahlquist DA: Molecular detection of pancreatic neoplasia: Current status and future promise. World J Gastroenterol 21: 11387-11395, 2015.

3. Gresham GK, Wells GA, Gill S, Cameron C and Jonker DJ: Chemotherapy regimens for advanced pancreatic cancer: A systematic review and network meta-analysis. BMC Cancer 14: 471, 2014.

4. de Sousa Cavalcante L and Monteiro G: Gemcitabine: Metabolism and molecular mechanisms of action, sensitivity and chemoresistance in pancreatic cancer. Eur J Pharmacol 741: 8-16, 2014.

5. Burris HA III, Moore MJ, Andersen J, Green MR, Rothenberg ML, Modiano MR, Cripps MC, Portenoy RK, Storniolo AM, Tarassoff P, et al: Improvements in survival and clinical benefit with gemcitabine as first-line therapy for patients with advanced pancreas cancer: A randomized trial. J Clin Oncol 15: 2403-2413, 1997.

6. Karnitz LM, Flatten KS, Wagner JM, Loegering D, Hackbarth JS, Arlander SJ, Vroman BT, Thomas MB, Baek YU, Hopkins KM, et al: Gemcitabine-induced activation of checkpoint signaling pathways that affect tumor cell survival. Mol Pharmacol 68 1636-1644, 2005.

7. Voutsadakis IA: Molecular predictors of gemcitabine response in pancreatic cancer. World J Gastrointest Oncol 3: 153-164, 2011.

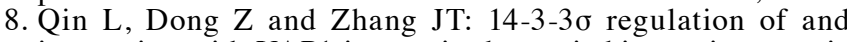
interaction with YAP1 in acquired gemcitabine resistance via promoting ribonucleotide reductase expression. Oncotarget 7: 17726-17736, 2016.
9. Maréchal A and Zou L: DNA damage sensing by the ATM and ATR kinases. Cold Spring Harb Perspect Biol 5: 5, 2013.

10. Couch FB, Bansbach CE, Driscoll R, Luzwick JW, Glick GG, Bétous R, Carroll CM, Jung SY, Qin J, Cimprich KA, et al: ATR phosphorylates SMARCAL1 to prevent replication fork collapse. Genes Dev 27: 1610-1623, 2013.

11. Sørensen CS and Syljuåsen RG: Safeguarding genome integrity: The checkpoint kinases ATR, CHK1 and WEE1 restrain CDK activity during normal DNA replication. Nucleic Acids Res 40: 477-486, 2012.

12. Buisson R, Boisvert JL, Benes $\mathrm{CH}$ and Zou L: Distinct but concerted roles of ATR, DNA-PK, and Chk1 in countering replication stress during S phase. Mol Cell 59: 1011-1024, 2015.

13. Jackson SP and Bartek J: The DNA-damage response in human biology and disease. Nature 461: 1071-1078, 2009.

14. Farmer H, McCabe N, Lord CJ, Tutt AN, Johnson DA, Richardson TB, Santarosa M, Dillon KJ, Hickson I, Knights C, et al: Targeting the DNA repair defect in $B R C A$ mutant cells as a therapeutic strategy. Nature 434: 917-921, 2005.

15. Kwok M, Davies N, Agathanggelou A, Smith E, Petermann E, Yates E, Brown J, Lau A and Stankovic T: Synthetic lethality in chronic lymphocytic leukaemia with DNA damage response defects by targeting the ATR pathway. Lancet 385 (Suppl 1): S58, 2015.

16. Fokas E, Prevo R, Hammond EM, Brunner TB, McKenna WG and Muschel RJ: Targeting ATR in DNA damage response and cancer therapeutics. Cancer Treat Rev 40: 109-117, 2014.

17. Weber AM and Ryan AJ: ATM and ATR as therapeutic targets in cancer. Pharmacol Ther 149: 124-138, 2015.

18. Prevo R, Fokas E, Reaper PM, Charlton PA, Pollard JR, McKenna WG, Muschel RJ and Brunner TB: The novel ATR inhibitor VE-821 increases sensitivity of pancreatic cancer cells to radiation and chemotherapy. Cancer Biol Ther 13: 1072-1081, 2012.

19. Fokas E, Prevo R, Pollard JR, Reaper PM, Charlton PA, Cornelissen B, Vallis KA, Hammond EM, Olcina MM, Gillies McKenna W, et al: Targeting ATR in vivo using the novel inhibitor VE-822 results in selective sensitization of pancreatic tumors to radiation. Cell Death Dis 3: e441, 2012.

20. Foote KM, Blades K, Cronin A, Fillery S, Guichard SS, Hassall L, Hickson I, Jacq X, Jewsbury PJ, McGuire TM, et al: Discovery of 4-\{4-[(3R)-3-Methylmorpholin-4-yl]-6-[1-(methylsulfonyl) cyclopropyl]pyrimidin-2-yl\}-1H-indole (AZ20): A potent and selective inhibitor of ATR protein kinase with monotherapy in vivo antitumor activity. J Med Chem 56: 2125-2138, 2013.

21. Nghiem P, Park PK, Kim Y, Vaziri C and Schreiber SL: ATR inhibition selectively sensitizes $G_{1}$ checkpoint-deficient cells to lethal premature chromatin condensation. Proc Natl Acad Sci USA 98: 9092-9097, 2001.

22. Mukhopadhyay UK, Senderowicz AM and Ferbeyre G: RNA silencing of checkpoint regulators sensitizes p53-defective prostate cancer cells to chemotherapy while sparing normal cells. Cancer Res 65: 2872-2881, 2005.

23. Toledo LI, Murga M, Zur R, Soria R, Rodriguez A, Martinez S, Oyarzabal J, Pastor J, Bischoff JR and Fernandez-Capetillo O: A cell-based screen identifies ATR inhibitors with synthetic lethal properties for cancer-associated mutations. Nat Struct Mol Biol 18: 721-727, 2011.

24. Ruzankina Y, Schoppy DW, Asare A, Clark CE, Vonderheide RH and Brown EJ: Tissue regenerative delays and synthetic lethality in adult mice after combined deletion of Atr and Trp53. Nat Genet 41: 1144-1149, 2009.

25. Wang G, Niu X, Zhang W, Caldwell JT, Edwards H, Chen W, Taub JW, Zhao L and Ge Y: Synergistic antitumor interactions between MK-1775 and panobinostat in preclinical models of pancreatic cancer. Cancer Lett 356: 656-668, 2015.

26. Xie C, Drenberg C, Edwards H, Caldwell JT, Chen W, Inaba H, Xu X, Buck SA, Taub JW, Baker SD, et al: Panobinostat enhances cytarabine and daunorubicin sensitivities in AML cells through suppressing the expression of BRCA1, CHK1, and Rad51. PLOS One 8: e79106, 2013.

27. Redon CE, Nakamura AJ, Zhang YW, Ji JJ, Bonner WM, Kinders RJ, Parchment RE, Doroshow JH and Pommier Y: Histone gammaH2AX and poly(ADP-ribose) as clinical pharmacodynamic biomarkers. Clin Cancer Res 16: 4532-4542, 2010.

28. Jossé R, Martin SE, Guha R, Ormanoglu P,Pfister TD, Reaper PM, Barnes CS, Jones J, Charlton P, Pollard JR, et al: ATR inhibitors VE-821 and VX-970 sensitize cancer cells to topoisomerase $i$ inhibitors by disabling DNA replication initiation and fork elongation responses. Cancer Res 74: 6968-6979, 2014. 
29. Kastan MB, Zhan Q, el-Deiry WS, Carrier F, Jacks T, Walsh WV Plunkett BS, Vogelstein B and Fornace AJ Jr: A mammalian cell cycle checkpoint pathway utilizing p53 and GADD45 is defective in ataxia-telangiectasia. Cell 71: 587-597, 1992.

30. D'Angiolella V, Donato V, Forrester FM, Jeong YT, Pellacani C, Kudo Y, Saraf A, Florens L, Washburn MP and Pagano M: Cyclin F-mediated degradation of ribonucleotide reductase M2 controls genome integrity and DNA repair. Cell 149: 1023-1034, 2012.

31. Jordheim LP, Sève P, Trédan O and Dumontet C: The ribonucleotide reductase large subunit (RRM1) as a predictive factor in patients with cancer. Lancet Oncol 12: 693-702, 2011.
32. Zhou J, Chen Z, Malysa A, Li X, Oliveira P, Zhang Y and Bepler G: A kinome screen identifies checkpoint kinase 1 (CHK1) as a sensitizer for RRM1-dependent gemcitabine efficacy. PLoS One 8: e58091, 2013.

33. Rahman MA, Amin ARMR, Wang D, Koenig L, Nannapaneni S, Chen Z, Wang Z, Sica G, Deng X, Chen ZG, et al: RRM2 regulates $\mathrm{Bcl}-2$ in head and neck and lung cancers: A potential target for cancer therapy. Clin Cancer Res 19: 3416-3428, 2013. 